\title{
Prognostic Accuracy of QSOFA Score Compared to SOFA Score among Patients with Sepsis
}

\author{
Hamsa B.T. ${ }^{1}$, Srinivas S.V. ${ }^{2}$, Prabhakar K. ${ }^{3}$, Maharaj L.S.Y.M. J. ${ }^{4}$, Raveesha A. ${ }^{5}$ \\ 1,2,3,4,5 Department of General Medicine, Sri Devraj Urs Academy of Higher \\ Education and Research, Kolar, Karnataka, India.
}

\section{ABSTRACT}

\section{BACKGROUND}

Sepsis is defined as life-threatening organ dysfunction caused by a dysregulated host response to infection. According to sepsis - 3 criteria, sepsis is diagnosed by Sequential organ failure assessment (SOFA) score of more than two. Surviving sepsis campaign introduced a newer scoring system, quick SOFA (QSOFA) score which uses only clinical parameters to prognosticate sepsis bed side and at the earliest. The purpose of this study was to evaluate the QSOFA score and then compare it to SOFA score in prognostication of sepsis.

\section{METHODS}

This study was a prospective observational study conducted in R. L. Jalappa Hospital among 150 individuals. Assessment of SOFA and QSOFA score was done and its significance in predicting mortality and morbidity was compared.

\section{RESULTS}

There were 87 males and 63 females. Mortality rate was $38.7 \%$. The initial QSOFA score of 1,2 and 3 had mortality rate of $5.2 \%, 24.1 \%$ and $70.7 \%$ respectively. Initial SOFA score of $<4,4-8$ and $>8$ had mortality rate of $5.2 \%, 37.9 \%$ and 56.9 $\%$ respectively. Interpretation - The SOFA score had statistically significant correlation in assessing need for ventilator support, QSOFA score had a significant relation assessing need for ventilator support, vasopressor support.

\section{CONCLUSIONS}

Both scores demonstrated good accuracy for predicting in-hospital mortality. The QSOFA scoring system can aid where the resources are limited.
Corresponding Author:

Dr. Hamsa B. T.,

Department of General Medicine,

Sri Devraj Urs Academy of Higher

Education and Research, Kolar,

Karnataka, India.

E-mail: hamsareddy12@gmail.com

DOI: $10.14260 / j e m d s / 2021 / 721$

How to Cite This Article:

Hamsa BT, Srinivas SV, Prabhakar K, et al. Prognostic accuracy of QSOFA score compared to SOFA score among patients with sepsis. J Evolution Med Dent Sci 2021;10(41):3557-3561,

10.14260/jemds/2021/721

Submission 03-07-2021,

Peer Review 20-09-2021,

Acceptance 28-09-2021,

Published 11-10-2021.

Copyright (c) 2021 Hamsa B.T. et al. This is an open access article distributed under Creative Commons Attribution License [Attribution 4.0 International (CC BY 4.0)]

\section{KEY WORDS}

Sepsis, SOFA Score, QSOFA Score, Septic Shock 


\section{BACKGROUND}

Sepsis is defined as life-threatening organ dysfunction caused by a dysregulated host response to infection.1,2 Similar to acute coronary syndrome, stroke and polytrauma, early identification and early initiation of goal directed management in the initial hours of sepsis improves outcome. ${ }^{1,3}$ Sepsis currently is one of the most common causes of mortality in the non-coronary intensive care units (ICU). 4,5

Sepsis is the most common cause for hospitalization in the worldwide. Patients often hospitalized for prolonged periods of up to 2 - 3 weeks. ${ }^{6}$ Despite the use of appropriate antimicrobial therapy and advanced supportive care, mortality in patients with sepsis has remained high since the past decade.7,8 Elderly subjects are especially vulnerable population and are susceptible to a wide array of infectious diseases. ${ }^{9}$

Cultures and serology results will be obtained only after 24 to 72 hours. In the initial hours of sepsis, we will determine the outcome and prognosis of sepsis patients. Hence, various guidelines propose the use of early empirical broad-spectrum antibiotics and supportive care, early recognition and treatment of complications, and intensive monitoring to prevent progression of organ dysfunction. ${ }^{3}$

According to sepsis - 3 criteria, the diagnosis of sepsis is mainly based on the change in SOFA score by 2 or more points consequent to the infection. ${ }^{1}$ SOFA score consists of 6 variables, which includes 2 clinical parameters and 4 laboratory values. In developing countries like India, with limited resource settings across the country, where rural population encounter primary care centers initially, lack of availability of laboratory facilities makes early prognostication of sepsis difficult according to SOFA score. Surviving sepsis campaign has also introduced a newer scoring system, the QSOFA score which uses clinical parameters alone to prognosticate sepsis bed side and at the earliest. QSOFA not only directs for early intensive management but also to take decisions regarding early referral to a tertiary care center from resource poor settings. ${ }^{1,10}$ Present study in evaluating the QSOFA score as prognostic marker in patients with sepsis when compared to SOFA score assumes more importance in lights of early identification and prognostication in resource poor settings.

\section{Objectives}

1. To assess the SOFA score in patients with sepsis

2. To assess the QSOFA score in patients with sepsis

3. To compare the above two scores with prognosis among subjects with sepsis.

\section{METHODS}

A prospective observational study was conducted including 150 patients satisfying the criteria for sepsis according to SOFA score of more than 2 (According to surviving sepsis campaign 3) who were admitted to medicine department of $\mathrm{R}$ L Jalappa Hospital, Tamaka, Kolar from November 2017 to September 2019.

\section{Inclusion Criteria}

Patients admitted to medicine department with sepsis. (According to the third international sepsis criteria: that is patients with SOFA score of $>2$ ) and patients aged more than 18 years.

\section{Exclusion Criteria}

Patients with pre-existing organ dysfunction prior to infection (chronic kidney disease, decompensated liver disease)

\section{Methodology}

Patients were included in the study after meeting inclusion and exclusion criteria. Detailed clinical examination and relevant investigations were done after obtaining written informed consent from patient/relative, patients were assessed for SOFA score and QSOFA score from day of admission, daily for 5 days/in hospital death. The need for supportive management was noted which included inotropic support, dialysis, ventilator support and ICU care. The outcome of the patient in terms of morbidity (length of ICU stay, need for ventilator support, inotropic support, and dialysis) and mortality was documented in terms of SOFA score and QSOFA score.

\section{Statistical Analysis}

Data was entered into Microsoft Excel data sheet and was analysed using Statistical Package for Social Sciences (SPSS 22 version software). Categorical data was represented in the form of frequencies and proportions. Chi square test or Fischer's exact test (for $2 \times 2$ tables only) was used as test of significance for qualitative data. Continuous data was represented as mean and standard deviation. Independent $t$ test was used as test of significance to identify the mean difference between two quantitative variables. SOFA and QSOFA score were further analysed using the receiver operating characteristic (ROC) and optimal cut-off points were chosen for the calculation of sensitivity, specificity. A test that predicts an outcome no better than chance has an area under the ROC curve of 0.5. An area under the ROC curve above 0.8 indicated fairly good prediction.

$P$ value (probability that the result is true) of $<0.05$ was considered as statistically significant after assuming all the rules of statistical tests.

\section{RESULTS}

This study was carried out in the period of November 2017 to September 2019 and 150 patients were studied. The subjects were in the age group of 20 to 95 years.

Of 150 patients of sepsis, 87 were male and 63 were females. Highest numbers of cases were in age group of 40 to 59 years i.e. 57 patients ( $38 \%$ ) followed by 60 to 79 years in 50 cases $(33.3 \%)$ (Mean \pm SD: $51.66 \pm$ 18.93). The commonest symptom in the study was fever which was seen in $76.7 \%$ of patients followed by cough (42\%), breathlessness (34.7\%), altered sensorium (30\%), vomiting 
(14\%), abdominal pain (8.7\%) and decreased urine output $(0.7 \%)$. Other less common symptoms were seizures, limb swelling, rashes and abdominal symptoms. Most common co morbidity was diabetes seen in $56 \%$ of study patients. Hypertension was next common seen in $32.67 \%$. $40 \%$ of study patients did not have any comorbidities. The most common diagnosis was lower respiratory tract infection (LRTI) seen in 71 (47.3\%) patients, 17 patients $(11.3 \%)$ with LRTI developed acute respiratory distress syndrome (ARDS), next common diagnosis was urosepsis seen in 22 $(14.7 \%)$ patients. Acute gastroenteritis was seen in 15 (10 $\%)$ patients, cellulitis in $13(8.7 \%)$ patients, neuro infection in $12(8 \%)$ patients.

Among 150 patients, 86 (57.3\%) needed ventilator support. 104 (69.3\%) patients required inotropic support. 20 $(13.4 \%)$ patients required renal replacement therapy due to sepsis induced acute kidney injury. Among 150 patients, 12 (8\%) patients required prolonged stay in ICU of more than 6 days, 72 (48\%) patients stayed for 3 - 6 days, 66 (44\%) patients needed less than 3 days. In the study, survivors were $92(61.3 \%)$, whereas non survivors were 58 (38.7\%). Among survivors, initial SOFA score was between 4 - 8 in 63 $\%$ patients, $<4$ in $26.1 \%$ patients and more than 8 in $10.9 \%$ patients, Figure 1
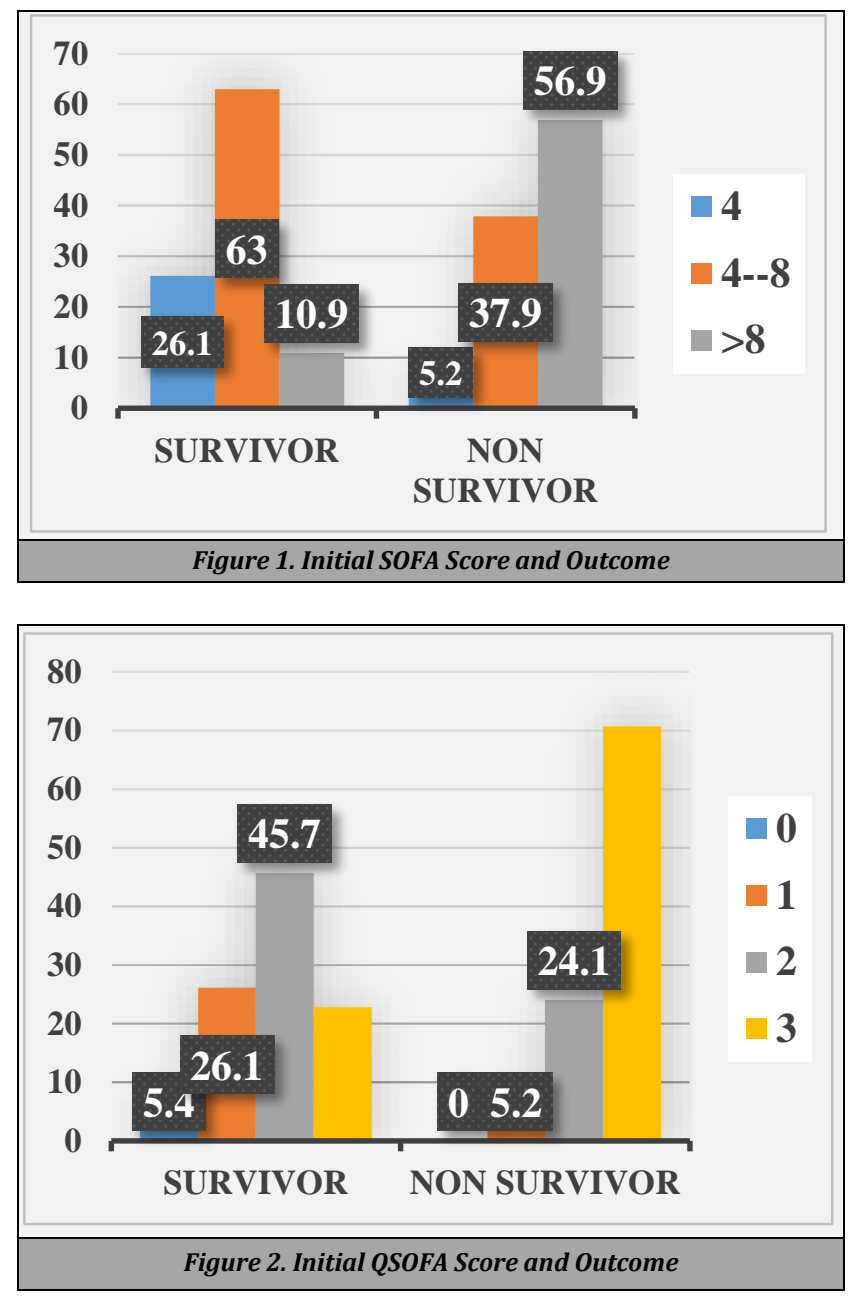

Among non-survivors, Initial SOFA score was between 4 8 in $37.9 \%$ patients, less than 4 in $5.2 \%$ patients and above 8 in $56.9 \%$ patients. $\mathrm{P}$ value $<0.001$, statistically significant difference found between initial SOFA score and outcome. Among survivors, initial QSOFA was 2 in $45.7 \%$ patients, 1 in
$26.1 \%$ patients, 3 in $22.8 \%$ patients and 0 in $5.4 \%$ patients. Figure 2. Among non-survivors, initial QSOFA score was 3 in $41.7 \%$ patients, 2 in $24.1 \%$ patients, 1 in 5.25 patients and none with QSOFA score of zero. P value $<0.001$, statistically significant difference was found between QSOFA score and outcome.

SOFA scores among non-survivors group was higher than survivors group. Significance was highest starting from day 3 and it remained significantly higher till day 5/last day in nonsurvivors. The mean SOFA score on day 1 among survivors was 5.39 (SD - 2.79) and among non survivors was 8.64 (SD3.6), and on day 5 the mean SOFA score in survivors was 3.22 (SD-2.38) and in non survivors was 10.33 (SD-3.58). The $P$ value was significant on all 5 days.

QSOFA score among non-survivors group was significantly higher than survivors group. Significance was highest starting from day 2 and it remained significantly higher till day 5/last day in non-survivors group. QSOFA score on day 1 in survivors was 1.86 and in non survivors, it was 2.66, and on day 5, the mean QSOFA score in survivors was 0.95 and in non survivors was 2.75. The $P$ value was significant on all 5 days.

Mean initial SOFA score in assessing requirement for ventilator support was significant with $P$ value $<0.001$. Whereas the mean initial SOFA score in assessing the requirement for inotropic support, haemodialysis and length of ICU stay was statistically not significant. Table 1.

\begin{tabular}{|c|c|c|c|c|}
\hline \multirow{2}{*}{ Morbidity Indicators } & & \multicolumn{2}{|c|}{ Sofa } & \multirow{2}{*}{ P Value } \\
\hline & & Mean & SD & \\
\hline \multirow{2}{*}{ Ventilator support } & No & 1.72 & .52 & \multirow{2}{*}{$<0.001$} \\
\hline & Yes & 2.40 & .64 & \\
\hline \multirow{2}{*}{ Inotropic support } & No & 1.93 & .65 & \multirow{2}{*}{0.040} \\
\hline & Yes & 2.18 & .68 & \\
\hline \multirow{2}{*}{ Haemodialysis } & No & 2.06 & .69 & \multirow{2}{*}{0.037} \\
\hline & Yes & 2.40 & .50 & \\
\hline \multirow{3}{*}{ Length of ICU stay } & $<3$ days & 2.14 & .78 & \multirow{3}{*}{0.805} \\
\hline & $3-6$ days & 2.07 & .59 & \\
\hline & $>6$ days & 2.17 & .58 & \\
\hline
\end{tabular}

The mean initial QSOFA score in assessing requirement for ventilator support and inotropic support was significant statistically with $\mathrm{P}$ value $<0.001$. Whereas initial mean QSOFA score in assessing the requirement for renal replacement therapy and length of ICU stay was statistically insignificant. Table 2

\begin{tabular}{|c|c|c|c|c|}
\hline \multirow{2}{*}{ Morbidity Indicators } & & \multicolumn{2}{|c|}{ QSOFA } & \multirow[b]{2}{*}{ P Value } \\
\hline & & Mean & SD & \\
\hline \multirow{2}{*}{ Ventilator support } & No & 1.81 & .91 & \multirow{2}{*}{$<0.001$} \\
\hline & Yes & 2.43 & .68 & \\
\hline \multirow{2}{*}{ Inotropic support } & No & 1.62 & .75 & \multirow{2}{*}{$<0.001$} \\
\hline & Yes & 2.40 & .77 & \\
\hline \multirow{2}{*}{ Renal replacement therapy } & No & 2.21 & .78 & \multirow{2}{*}{0.127} \\
\hline & Yes & 1.90 & 1.12 & \\
\hline \multirow{3}{*}{ Length of ICU stay } & $<3$ days & 2.26 & .79 & \multirow{3}{*}{0.287} \\
\hline & $3-6$ days & 2.06 & .87 & \\
\hline & $>6$ days & 2.33 & .89 & \\
\hline
\end{tabular}

An area under the receiver operating characteristics (ROC) curve above 0.8 indicated fairly good prediction. Area under the ROC curve for both SOFA and QSOFA score was almost similar with 0.767 and 0.757 respectively, suggesting that they are similar in assessing outcome (mortality). SOFA score on day 1 had a sensitivity of $56.9 \%$ and specificity of $89.1 \%$ in predicting mortality, and QSOFA score on day 1 had 
a sensitivity of $70.7 \%$ and specificity of $77.2 \%$ in predicting mortality. Table 3

\begin{tabular}{|ccc|}
\hline & QSOFA Score & SOFA Score \\
Area under the ROC curve (AUC) & 0.767 & 0.757 \\
Standard error & 0.0360 & 0.0411 \\
95 \% confidence interval & 0.691 to 0.832 & 0.681 to 0.824 \\
P value & $<0.0001$ & $<0.0001$ \\
\hline Table 3. Area Under the ROC Curve of QSOFA and SOFA Score \\
\hline
\end{tabular}
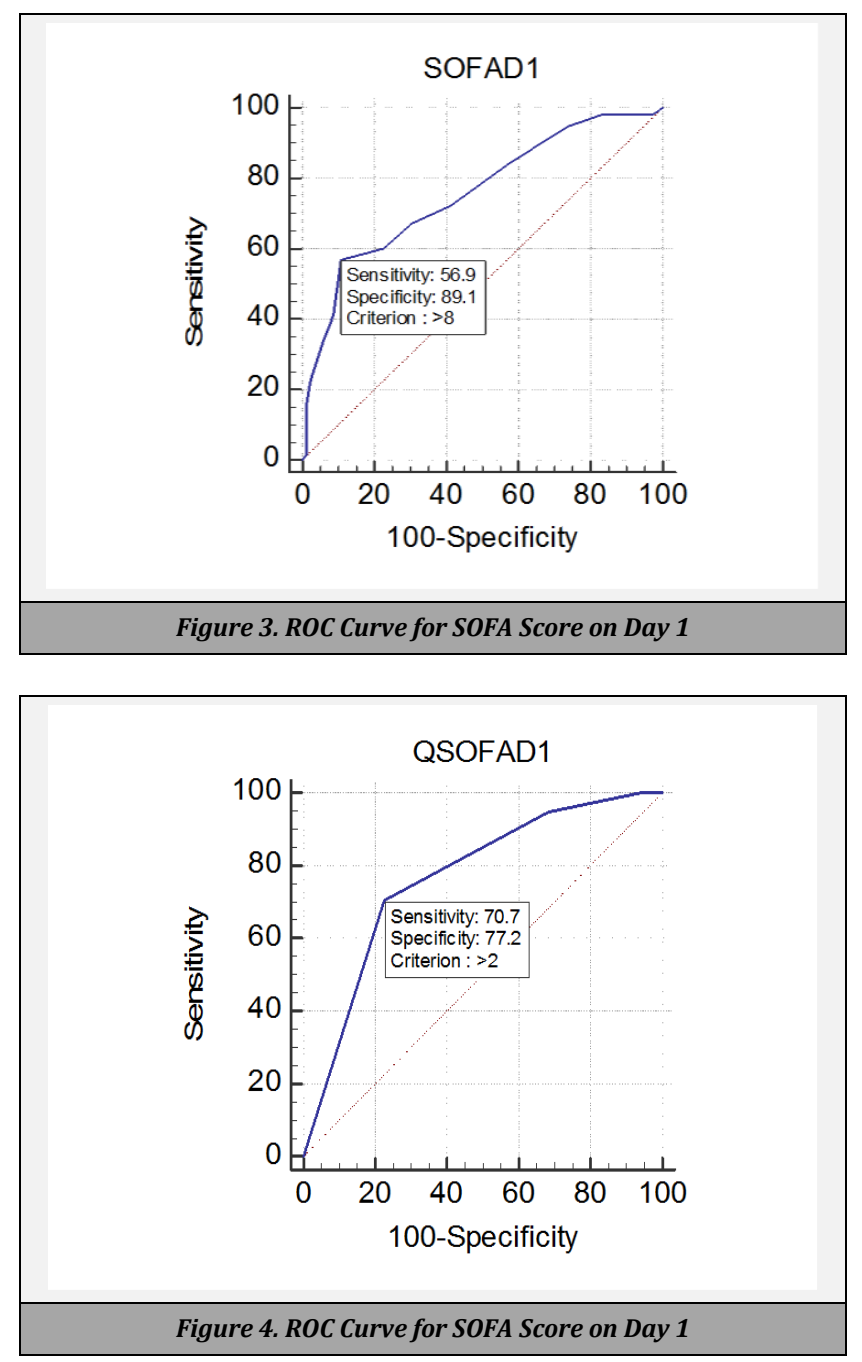

\section{DISCUSSION}

The study included 87 males and 63 females win the age group between 18 years to 95 years. Mean age in the study was 51.66 years. Male preponderance has seen in similar studies in India. 687 were male and 63 females in this study.

Most common symptom in our patients was fever, followed by cough, breathlessness, altered sensorium, pain abdomen and reduced urine output. Reduced urine output was observed in 29 patients of acute kidney injury (AKI). Among various organ dysfunctions in sepsis, AKI is the most morbid condition since it independently increases of mortality, as well as it increases cost of care. ${ }^{11}$

Proportion of patients with SOFA score of $<4$ on day 1 in our study was similar to the study done by Ferreira FL et al.12 whereas it was higher in study by Hewett et al. ${ }^{13}$ SOFA score between 4 - 8 was higher in this study when compared to above two studies. In this study SOFA score of $>8$ on the day of presentation was seen in $28.7 \%$ patients suggesting significant multi-organ dysfunction at the time of presentation.

Mortality of $38.7 \%$ is noted in this study.in studies done by Abhinandhan et al. ${ }^{14}$ and Rachel Oommen et al. ${ }^{15}$ reported a mortality of $36 \%$ and $34 \%$ respectively, similar to the present study. Mortality in sepsis ranges between $13 \%$ and $50 \%$ in numerous large clinical trials.

Respiratory infection was the most common cause of sepsis in the study, 17 patients with pneumonia progressed to ARDS. 22 cases of urinary tract infection (UTI) associated septicaemia was observed. 13 patients had cellulitis. 12 had meningitis and 15 patients had gastroenteritis with sepsis.

Organ dysfunction and need for supportive care: In the current study, requirement for ventilator support was seen in $86(57.3 \%)$ patients, the mean SOFA score and mean QSOFA score of these patients were 2.4 and 2.43 respectively, both were statistically significant with $\mathrm{P}$ value of $<0.001$. $42.7 \%$ patients did not require ventilator support.

Requirement for vasopressor therapy was noted in 104 (69.3\%) patients among whom the mean SOFA and QSOFA score was 1.93 and 1.62 respectively, mean SOFA score in assessing need for vasopressor therapy was statistically insignificant whereas for mean QSOFA score it was significant statistically with $P$ value of $<0.001$. Requirement for haemodialysis due to sepsis related AKI was seen in 20 (13.4\%), in whom the mean SOFA and QSOFA score was 2.4 and 1.90 respectively, both the mean SOFA and QSOFA in assessing need for haemodialysis was statistically insignificant with $\mathrm{p}$ value of 0.037 and 0.127 respectively. Majority of the patients in the study did not develop AKI.

Based on length of ICU stay, patients were divided into three groups, those who required ICU care for $<3$ days were $66(44 \%)$ patients in those the mean SOFA and QSOFA score was 2.4 and 2.26 respectively. Those who stayed between 3 6 days were 72 (48\%) patients, in them the mean SOFA and QSOFA score was 2.07 and 2.06 respectively. 12 (8\%) patients stayed for more than 6 days in them the mean SOFA and QSOFA score was 2.17 and 2.33 respectively. For the assessment of duration of ICU stay, the P value for both mean SOFA and QSOFA score was statistically insignificant with $\mathrm{P}$ values of 0.805 and 0.283 respectively.

\section{Predictors of Mortality}

In the current study, 58 patients succumbed and 92 patients survived. Among non-survivors, the mean age was little high when compared to survivors (54.42 v/s 48.90) which was statistically insignificant $(\mathrm{P}=0.146)$.

When compared to study done by Abhinandhan et al.14 current study had statistically significant correlation on all 5 days, whereas in the study quoted above statistically significant correlation was seen only on day 3 and 5 .

Mean SOFA score of above 7 had $87 \%$ mortality predictive value. Highest SOFA score of 11 had $85.7 \%$ mortality predictive value.in study by Acharya et al. ${ }^{9}$ and Ferreira FL et al. ${ }^{12}$ had a mortality predictive value of $87.5 \%$ and $85 \%$ respectively with highest SOFA score of above 11 .

In the current study, mortality with initial QSOFA score of 0 was $0 \%$, in a study by Rudd, Kristina et al. ${ }^{16}$ the mortality with QSOFA of 0 was $3 \%$ and in their study, QSOFA score of 
3 was associated with only $30 \%$ mortality whereas current study has $70.7 \%$ mortality with similar score.

Area under the ROC curve above 0.8 indicated fairly good prediction. Our study had an AUC of 0.767 compared to study by Rudd et al. ${ }^{17}$ who had an AUC of 0.69 .

\section{CONCLUSIONS}

Sepsis carries a high mortality rate. In our study, it was 38.7. LRTI is the most frequent cause for severe sepsis in developing countries like India. Prompt identification of patients at risk for developing sepsis and classifying them with QSOFA score at bedside with only clinical variables helps in priority care to such patients who are at increased risk.

QSOFA score and the SOFA score demonstrated fair to good accuracy for predicting in-hospital mortality when implicated to patients with severe sepsis. The initial QSOFA score of 1, 2 and 3 had $5.2 \%, 24.1 \%$ and $70.7 \%$ mortality rate respectively. Initial SOFA score of $<4,4-8$ and $>8$ had mortality rate of $5.2 \%, 37.9 \%$ and $56.9 \%$ respectively.

The mean SOFA score had statistically significant correlation with respect to assessment of ARDS and subsequent ventilator support whereas the mean QSOFA score had a statistically significant relation in predicting need for ventilator support and vasopressor support. Both the scores had statistically insignificant correlation with respect to assessment of AKI and need for haemodialysis and in predicting the probable length of ICU care.

The QSOFA scoring system can aid the physicians in early referral to health care centre, in admitting patients to ICU, monitoring the clinical course, assessing organ dysfunction, prediction of mortality, and for transferring patients out of ICU and hence in proper utilization of ICU resources in developing countries.

Data sharing statement provided by the authors is available with the full text of this article at jemds.com.

Financial or other competing interests: None.

Disclosure forms provided by the authors are available with the full text of this article at jemds.com.

\section{REFERENCES}

[1] Singer M, Deutschman CS, Seymour CW, et al. The third international consensus definitions for sepsis and septic shock. J Am Med Assoc 2016;315(8):801-10.

[2] Abraham E. New definitions for sepsis and septic shock continuing evolution but with much still to be done. J Am Med Assoc 2015;315(8):757-9.
[3] Dellinger RP, Levy MM, Rhodes A, et al. Surviving sepsis campaign: international guidelines for management of severe sepsis and septic shock. Intensive Care Med 2013;39(2):165-228.

[4] Levy MM, Evans LE, Rhodes A. The surviving sepsis campaign bundle: 2018 update. Intensive Care Med 2018;44(6):925-8.

[5] Balk RA. Severe sepsis and septic shock: definitions, epidemiology, and clinical manifestations. Crit Care Clin 2000;16(2):179-92.

[6] Balk RA. Optimum treatment of severe sepsis and septic shock: evidence in support of the recommendations. Dis Mon 2004;50(4):168-213.

[7] Iwashyna TJ, Ely EW, Smith DM, et al. Long-term cognitive impairment and functional disability among survivors of severe sepsis. J Am Med Assoc 2010;304(16):1787-94.

[8] Degoricija V, Sharma M, Legac A, et al. Survival analysis of 314 episodes of sepsis in medical intensive care unit in university hospital: impact of intensive care unit performance and antimicrobial therapy. Croat Med J 2006;47(3):385-97.

[9] Acharya SP, Pradhan B, Marhatta MN. Application of "the Sequential Organ Failure Assessment (SOFA) score in predicting outcome in ICU patients with SIRS. Kathmandu Univ Med J 2007;5(4):475-83.

[10] Adhikari NKJ, Rubenfeld GD. qSOFA score for patients with sepsis in low - and middle - income countries. JAMA 2018;319(21):2175-7.

[11] Ronco C, Kellum JA, Bellomo R, et al. Potential interventions in sepsis-related acute kidney injury. Clin J Am Soc Nephrol 2008;3(2):531-44.

[12] Ferreira FL, Bota DP, Bross A, et al. Serial evaluation of the SOFA scores to predict outcome in critically ill patients. J Am Med Assoc 2001;286(14):1754-8.

[13] Hewett JN, Rodgers GW, Chase JG, et al. Assessment of SOFA score as a diagnostic indicator in intensive care medicine. The International Federation of Automatic Control 2012;8(1):467-71.

[14] Abhinandan KS, Vedavathi R. Usefulness of sequential organ failure assessment (sofa) and acute physiology and chronic health evaluation ii (apache ii) score in analysing patients with multiple organ dysfunction syndrome in sepsis. Journal of Evolution of Medical and Dental Sciences 2013;2(49):9591-605.

[15] Rachel Oommen SP. Clinical and microbiological profile of sepsis in patients admitted to medicine ward in kvg medical college and hospital. Indian J Med Res 2014;159(4):459-68.

[16] Rudd KE, Seymour CW, Aluisio AR, et al. Association of the Quick Sequential (Sepsis - Related) Organ Failure Assessment (QSOFA) score with excess hospital mortality in adults with suspected infection in low - and middle-income countries. JAMA 2018;319(21):2202-11. 